1 Hacettepe Journal of Mathematics and Statistics

$\bigcap$ Volume 45 (4) (2016), 1033-1047

\title{
On quasi-contractions in metric spaces with a graph
}

\author{
Kamal Fallahi* and Aris Aghanians ${ }^{\dagger \ddagger}$
}

\begin{abstract}
In the present work, we introduce $G$-quasi-contractions using directed graphs in metric spaces with a graph and we show that this contraction generalizes a large number of contractions. We then investigate the existence of fixed points for $G$-quasi-contractions under two different conditions and discuss the main theorem. Finally, we list some consequences of our theorem where either the contractive condition is replaced with a stronger one or the underlying space is changed to a complete metric space or a complete cone metric space.
\end{abstract}

Keywords: ( $\widetilde{\mathrm{C}})$-Graph, $\widetilde{G}$-Quasi-contraction, Fixed point.

2000 AMS Classification: 47H10, 05C40

Received : 22.05.2015 Accepted : 20.10.2015 Doi : 10.15672/HJMS.20164514282

\section{Introduction and Preliminaries}

In 1974, Lj. B. Ćirić [9] introduced (single-valued) quasi-contractions in metric spaces and gave an example to show that this new contraction is a real generalization of some well-known linear contractions. He investigated the existence and uniqueness of fixed points for quasi-contractions in $T$-orbitally complete metric spaces via a different approach rather than using merely the iterates of a point. He also introduced multi-valued quasi-contractions and showed that a similar result is valid for these contractions in $F$-orbitally complete metric spaces.

In [21], B. E. Rhoades compared various definitions of contractive mappings in metric spaces and showed that Cirić's contractive condition is one of the most general contractive definitions in metric spaces and includes a large number of different types of contractions. Thus, many authors became interested in studying quasi-contractions. The existence and uniqueness of fixed points for these contractions as well as some interesting properties

*Department of Mathematics, Payame Noor University, P.O. Box 19395-3697, Tehran, Iran, Email: fallahi1361@gmail.com

${ }^{\dagger}$ Department of Mathematics, Payame Noor University, P.O. Box 19395-3697, Tehran, Iran, Email: a.aghanians@dena.kntu.ac.ir

$\ddagger$ Corresponding Author. 
of them have been investigated not only in metric spaces but in different spaces such as modular spaces (see, e.g., [17]) and cone metric spaces (see, e.g., [13, 15, 16, 20]) so far. Quasi-contractions have also been studied in Banach spaces (see, e.g., [10]).

The most important graph theory approach to metric fixed point theory introduced so far is attributed to J. Jachymski [14]. In this approach, the underlying metric space is equipped with a directed graph and the Banach contraction is formulated in a graph language. Using this simple but very interesting idea, J. Jachymski generalized several well-known versions of Banach contraction principle in metric spaces simultaneously and from various aspects. As an application, he proved the Kelisky-Rivlin theorem on the iterates of the Bernstein operators defined on the Banach space of continuous functions on $[0,1]$. In the recent years, many authors followed J. Jachymski's idea to formulate different types of contractions via directed graphs in metric spaces and generalized the concerned fixed point theorems (see, e.g. [1, 2, 3, 6]).

The main goal of this paper is to formulate single-valued quasi-contractions in metric spaces with a graph and find sufficient conditions which guarantee the existence of a fixed point. A large number of different types of contractive mappings formulated using directed graphs satisfy the presented contractive condition and our main result is a natural generalization of [9, Theorem 1] from metric spaces to metric spaces with a graph.

We start by reviewing a few basic notions in graph and fixed point theory that are frequently used in the paper. For more details on graphs, the reader is refered to [4].

In an arbitrary (not necessarily simple) graph $G$, a link is an edge of $G$ with distinct ends and a loop is an edge of $G$ with identical ends. Two or more links of $G$ with the same pairs of ends are called parallel edges of $G$.

Suppose that $(X, d)$ is a metric space and $G$ is a directed graph whose vertex set $V(G)$ coincides with $X$ and edge set $E(G)$ contains all loops (note that in general, $G$ can have uncountably many vertices). Suppose further that $G$ has no parallel edges. In this case, $(X, d)$ is called a metric space with the graph $G$.

By $G^{-1}$, it is meant the conversion of $G$ as usual, i.e. a directed graph obtained from $G$ by reversing the directions of the edges of $G$, and by $\widetilde{G}$, it is always meant the undirected graph obtained from $G$ by ignoring the directions of the edges $G$. Thus, it is clear that $V\left(G^{-1}\right)=V(\widetilde{G})=V(G)=X$ and we have

$$
E\left(G^{-1}\right)=\{(x, y) \in X \times X:(y, x) \in E(G)\} \quad \text { and } \quad E(\widetilde{G})=E(G) \cup E\left(G^{-1}\right) .
$$

If $(X, \preccurlyeq)$ is a partially ordered set, then by comparable elements of $(X, \preccurlyeq)$, it is meant two elements $x, y \in X$ satisfying either $x \preccurlyeq y$ or $y \preccurlyeq x$, and following A.C. M. Ran and M. C. B. Reurings [19, Theorem 2.1], a mapping $T: X \rightarrow X$ is called order-preserving whenever $x \preccurlyeq y$ implies $T x \preccurlyeq T y$ for all $x, y \in X$. Furthermore, following the idea of A. Petruşel and I. A. Rus in L-spaces [18, Definitions 3.1 and 3.6] (see also [23]), one can naturally formulate Picard and weakly Picard operators in metric spaces as follows:

1.1. Definition $([14,18,23])$. Let $(X, d)$ be a metric space and $T: X \rightarrow X$ be a mapping.

a) $T$ is called a Picard operator if $T$ has a unique fixed point $x^{\star} \in X$ and $T^{n} x \rightarrow x^{\star}$ for all $x \in X$.

b) $T$ is called a weakly Picard operator if $\left\{T^{n} x\right\}$ is a convergent sequence and its limit (which depends on $x$ ) is a fixed point of $T$ for all $x \in X$.

Finally, we need a weaker type of continuity defined in metric spaces with a graph which was first introduced by J. Jachymski (see [14, Definition 2.4]). The idea of this definition comes from the definition of orbital continuity defined by $\mathrm{Lj}$. B. Ćirić [8].

1.2. Definition $([14])$. Let $(X, d)$ be a metric space with a graph $G$. A mapping $T$ : $X \rightarrow X$ is called orbitally $G$-continuous on $X$ if $T^{b_{n}} x \rightarrow y$ implies $T\left(T^{b_{n}} x\right) \rightarrow T y$ for 
all $x, y \in X$ and all sequences $\left\{b_{n}\right\}$ of positive integers such that $\left(T^{b_{n}} x, T^{b_{n}+1} x\right) \in E(G)$ for all $n \in \mathbb{N}$.

\section{Main Results}

Let $(X, d)$ be a metric space with a graph $G$ and let $T: X \rightarrow X$ be a mapping. In this section, by $C_{T}$, we mean the set of all points $x \in X$ such that $\left(T^{m} x, T^{n} x\right)$ is an edge of $\widetilde{G}$ for all $m, n \in \mathbb{N} \cup\{0\}$, i.e.

$$
C_{T}=\left\{x \in X:\left(T^{m} x, T^{n} x\right) \in E(\widetilde{G}) \quad m, n=0,1, \ldots\right\} .
$$

Note that $C_{T}$ may be an empty set. For instance, consider the set $\mathbb{R}$ of all real numbers with the usual Euclidean metric and a graph $G$ given by $V(G)=\mathbb{R}$ and $E(G)=\{(x, x)$ : $x \in \mathbb{R}\}$. If $T: \mathbb{R} \rightarrow \mathbb{R}$ is defined by the rule $T x=x+1$ for all $x \in \mathbb{R}$, then it is easily seen that $C_{T}=\emptyset$.

Given $x \in X$ and $n \in \mathbb{N} \cup\{0\}$, the $n$-th orbit of $x$ under $T$ is denoted by $O(x ; n)$, i.e.

$$
O(x ; n)=\left\{x, T x, \ldots, T^{n} x\right\} .
$$

Finally, if $A$ is a subset of $X$, then by $\operatorname{diam}(A)$, it is meant the diameter of $A$ in $X$, i.e.

$$
\operatorname{diam}(A)=\sup \{d(x, y): x, y \in A\} .
$$

Following the idea of S. M. A. Aleomraninejad et al. [1], we say that $G$ is a $(\widetilde{\mathrm{C}})$-graph whenever the triple $(X, d, G)$ has the following property:

If $x \in X$ and $\left\{x_{n}\right\}$ is a sequence in $(X, d, G)$ such that $x_{n} \rightarrow x$ and $\left(x_{n}, x_{n+1}\right) \in$ $E(\widetilde{G})$ for all $n \in \mathbb{N}$, then there exists a subsequence $\left\{x_{n_{k}}\right\}$ of $\left\{x_{n}\right\}$ such that $\left(x_{n_{k}}, x\right) \in E(\widetilde{G})$ for all $k \in \mathbb{N}$.

Now, we are ready to give the definition of $G$-quasi-contractions in metric spaces with a graph which is motivated by [9, Definition 1] and [14, Definition 2.1].

2.1. Definition. Let $(X, d)$ be a metric space with a graph $G$ and $T: X \rightarrow X$ be a mapping. We say that $T$ a $G$-quasi-contraction if

Q1) $T$ preserves the edges of $G$, i.e. $(x, y) \in E(G)$ implies $(T x, T y) \in E(G)$ for all $x, y \in X$

Q2) there exists a $\lambda \in[0,1)$ such that

$$
d(T x, T y) \leq \lambda \cdot \max \{d(x, y), d(x, T x), d(y, T y), d(x, T y), d(y, T x)\}
$$

for all $x, y \in X$ with $(x, y) \in E(G)$.

We also call the number $\lambda$ in (Q2) a quasi-contractive constant of $T$.

We now give some examples of $G$-quasi-contractions.

2.2. Example. Suppose that $(X, d)$ is a metric space with a graph $G$ and $x_{0} \in X$. It is easy to verify that the constant mapping $x \mapsto x_{0}$ is a $G$-quasi-contraction. So the cardinality of the set of all $G$-quasi-contractions defined on a metric space $(X, d)$ with a graph $G$ is no less than the cardinality of $X$.

2.3. Example. Suppose that $(X, d)$ is a metric space and $T: X \rightarrow X$ is a quasicontraction in the sense of $\mathrm{Lj}$. B. Ćirić [9, Definition 1], i.e. there exists a $\lambda \in[0,1)$ such that

$$
d(T x, T y) \leq \lambda \cdot \max \{d(x, y), d(x, T x), d(y, T y), d(x, T y), d(y, T x)\}
$$

for all $x, y \in X$. Define a graph $G_{0}$ by $V\left(G_{0}\right)=X$ and $E\left(G_{0}\right)=X \times X$, i.e. $G_{0}$ is the complete graph whose vertex set coincides with $X$. Clearly, $T$ preserves the edges of $G_{0}$ and (2.1) guarantees that $T$ satisfies (Q2) for the complete graph $G_{0}$. Thus, $T$ is a 
$G_{0}$-quasi-contraction. Hence $G_{0}$-quasi-contractions on metric spaces with the graph $G_{0}$ are precisely the quasi-contractions on metric spaces, and so $G$-quasi-contractions are a generalization of quasi-contractions from metric spaces to metric spaces with a graph.

2.4. Example. Suppose that $(X, \preccurlyeq)$ is a partially ordered set and $d$ is a metric on $X$. Define a graph $G_{1}$ by $V\left(G_{1}\right)=X$ and $E\left(G_{1}\right)=\{(x, y) \in X \times X: x \preccurlyeq y\}$. A mapping $T: X \rightarrow X$ preserves the edges of $G_{1}$ if and only if $T$ is order-preserving, and $T$ satisfies (Q2) for the graph $G_{1}$ if and only if there exists a $\lambda \in[0,1)$ such that

$$
d(T x, T y) \leq \lambda \cdot \max \{d(x, y), d(x, T x), d(y, T y), d(x, T y), d(y, T x)\}
$$

for all comparable elements $x, y \in X$.

2.5. Example. Suppose that $(X, \preccurlyeq)$ is a partially ordered set and $d$ is a metric on $X$. Define a graph $G_{2}$ by $V\left(G_{2}\right)=X$ and $E\left(G_{2}\right)=\{(x, y) \in X \times X: x \preccurlyeq y \vee y \preccurlyeq x\}$. A mapping $T: X \rightarrow X$ preserves the edges of $G_{2}$ if and only if $T$ maps comparable elements of $(X, \preccurlyeq)$ onto comparable elements, and $T$ satisfies (Q2) for the graph $G_{2}$ if and only if there exists a $\lambda \in[0,1)$ such that

$$
d(T x, T y) \leq \lambda \cdot \max \{d(x, y), d(x, T x), d(y, T y), d(x, T y), d(y, T x)\}
$$

for all comparable elements $x, y \in X$. In particular, if $T$ is a $G_{1}$-quasi-contraction, then $T$ is a $G_{2}$-quasi-contraction. Hence $G$-quasi-contractions are a generalization of ordered quasi-contractions from metric spaces equipped with a partial order to metric spaces with a graph.

2.6. Example. Suppose that $(X, d)$ is a metric space and $\varepsilon>0$ is a fixed real number. Recall that two elements $x, y \in X$ are said to be $\varepsilon$-close if $d(x, y)<\varepsilon$. Define a graph $G_{3}$ by $V\left(G_{3}\right)=X$ and $E\left(G_{3}\right)=\{(x, y) \in X \times X: d(x, y)<\varepsilon\}$. A mapping $T: X \rightarrow X$ preserves the edges of $G_{3}$ if and only if $T$ maps $\varepsilon$-close elements of $(X, d)$ onto $\varepsilon$-close elements, and $T$ satisfies (Q2) for the graph $G_{3}$ if and only if there exists a $\lambda \in[0,1)$ such that

$$
d(T x, T y) \leq \lambda \cdot \max \{d(x, y), d(x, T x), d(y, T y), d(x, T y), d(y, T x)\}
$$

for all $\varepsilon$-close elements $x, y \in X$.

Hereafter, we assume that the graphs $G_{0}, G_{1}, G_{2}$ and $G_{3}$ are as defined in Examples $2.3,2.4,2.5$ and 2.6 , respectively.

2.7. Remark. In the definitions of $(\widetilde{\mathrm{C}})$-graph and the set $C_{T}$, let's set $G$ the special graphs $G_{0}, G_{1}, G_{2}$ and $G_{3}$. Then we obtain the following special cases:

- The set $C_{T}$ related to the complete graph $G_{0}$ coincides with $X$ and $G_{0}$ is a $(\widetilde{\mathrm{C}})$-graph.

- If $\preccurlyeq$ is a partial order on $X$, then the set $C_{T}$ related to the graph $G_{1}$ (and also $G_{2}$ ) consists of all points $x \in X$ whose every two iterates under $T$ are comparable elements of $(X, \preccurlyeq)$. In addition, $G_{1}$ (and also $G_{2}$ ) is a $(\widetilde{\mathrm{C}})$-graph whenever the triple $(X, d, \preccurlyeq)$ has the following property:

$(*)$ If $\left\{x_{n}\right\}$ is a sequence in $(X, d)$ converging to an $x \in X$ whose successive terms are pairwise comparable elements of $(X, \preccurlyeq)$, then there exists a subsequence of $\left\{x_{n}\right\}$ whose terms and $x$ are comparable elements of $(X, \preccurlyeq)$.

- If $\varepsilon>0$, then the set $C_{T}$ relative to the graph $G_{3}$ consists of all points $x \in X$ whose every two iterates under $T$ are $\varepsilon$-close elements of $(X, d)$. In addition, $G_{3}$ is a $(\widetilde{\mathrm{C}})$-graph. Indeed, if $\left\{x_{n}\right\}$ is a sequence in $(X, d)$ converging to an $x \in X$, then for sufficiently large indices $n$, say $n \geq N$, we have $d\left(x_{n}, x\right)<\varepsilon$. Therefore, $\left\{x_{n+N}\right\}$ is a subsequence of $\left\{x_{n}\right\}$ whose terms and $x$ are $\varepsilon$-close elements of $(X, d)$. 
2.8. Example. Suppose that $(X, d)$ is a metric space with a graph $G$ and $T: X \rightarrow X$ is a Banach $G$-contraction in the sense of J. Jachymski [14, Definition 2.1], i.e. $T$ preserves the edges of $G$ and there exists an $\alpha \in(0,1)$ such that

$$
d(T x, T y) \leq \alpha d(x, y)
$$

for all $x, y \in X$ with $(x, y) \in E(G)$. If $(x, y) \in E(G)$, then

$$
d(T x, T y) \leq \alpha d(x, y) \leq \alpha \cdot \max \{d(x, y), d(x, T x), d(y, T y), d(x, T y), d(y, T x)\} .
$$

Therefore, $T$ satisfies (Q2) and so $T$ is a $G$-quasi-contraction. Hence every $G$-contraction is a $G$-quasi-contraction.

2.9. Example. Suppose that $(X, d)$ is a metric space with a graph $G$ and $T: X \rightarrow X$ is a $G$-Kannan mapping in the sense of F. Bojor [2, Definition 4], i.e. $T$ preserves the edges of $G$ and there exists an $\alpha \in\left[0, \frac{1}{2}\right)$ such that

$$
d(T x, T y) \leq \alpha(d(x, T x)+d(y, T y))
$$

for all $x, y \in X$ with $(x, y) \in E(G)$. If $(x, y) \in E(G)$, then

$$
\begin{aligned}
d(T x, T y) & \leq \alpha(d(x, T x)+d(y, T y)) \\
& \leq 2 \alpha \cdot \max \{d(x, T x), d(y, T y)\} \\
& \leq 2 \alpha \cdot \max \{d(x, y), d(x, T x), d(y, T y), d(x, T y), d(y, T x)\}
\end{aligned}
$$

Therefore, $T$ satisfies (Q2) and so $T$ is a $G$-quasi-contraction. Hence every $G$-Kannan mapping is a $G$-quasi-contraction.

2.10. Example. Suppose that $(X, d)$ is a metric space with a graph $G$ and $T: X \rightarrow X$ is a $G$-Chatterjea mapping in the sense that $T$ preserves the edges of $G$ and there exists an $\alpha \in\left[0, \frac{1}{2}\right)$ such that

$$
d(T x, T y) \leq \alpha(d(x, T y)+d(y, T x))
$$

for all $x, y \in X$ with $(x, y) \in E(G)$ (see [5, 21] for the definition in metric spaces). If $(x, y) \in E(G)$, then an argument similar to that appeared in Example 2.9 establishes that

$$
d(T x, T y) \leq 2 \alpha \cdot \max \{d(x, y), d(x, T x), d(y, T y), d(x, T y), d(y, T x)\}
$$

Therefore, $T$ satisfies (Q2) and so $T$ is a $G$-quasi-contraction. Hence every $G$-Chatterjea mapping is a $G$-quasi-contraction.

2.11. Example. Suppose that $(X, d)$ is a metric space with a graph $G$ and $T: X \rightarrow X$ is a $G$-Ćirić-Reich-Rus operator in the sense of F. Bojor [3, Definition 7], i.e. $T$ preserves the edges of $G$ and there exist $a, b, c \geq 0$ with $a+b+c<1$ such that

$$
d(T x, T y) \leq a d(x, y)+b d(x, T x)+c d(y, T y)
$$

for all $x, y \in X$ with $(x, y) \in E(G)$. If $(x, y) \in E(G)$, then an argument similar to that appeared in Example 2.9 establishes that

$$
d(T x, T y) \leq(a+b+c) \cdot \max \{d(x, y), d(x, T x), d(y, T y), d(x, T y), d(y, T x)\} .
$$

Therefore, $T$ satisfies (Q2) and so $T$ is a $G$-quasi-contraction. Hence every $G$-Ćirić-ReichRus operator is a $G$-quasi-contraction.

Now, suppose that $T: X \rightarrow X$ is a Ćirić-Reich-Rus $G$-contraction in the sense of C. Chifu and G. Petruşel [6, Definition 2.2], i.e. $T$ preserves the edges of $G$ and there exist $\alpha, \beta, \gamma>0$ with $\alpha+\beta+\gamma<1$ such that

$$
d(T x, T y) \leq \alpha d(x, y)+\beta d(x, T x)+\gamma d(y, T y)
$$


for all $x, y \in X$ with $(x, y) \in E(G)$. Then by a similar argument, one can easily see that $T$ is a $G$-quasi-contraction. Hence every Cirić-Reich-Rus $G$-contraction is a $G$-quasicontraction.

2.12. Example. Suppose that $(X, d)$ is a metric space and $T: X \rightarrow X$ is a $\lambda$-generalized contraction in the sense of $\mathrm{Lj}$. B. Ćirić [7, Definition 2.1], i.e. for all $x, y \in X$, there exist four functions $q, r, s, t: X \times X \rightarrow[0, \infty)$ with

$$
\sup \{q(x, y)+r(x, y)+s(x, y)+2 t(x, y): x, y \in X \times X\}=\lambda<1
$$

such that

$$
\begin{aligned}
d(T x, T y) \leq & q(x, y) d(x, y)+r(x, y) d(x, T x)+s(x, y) d(y, T y) \\
& +t(x, y)(d(x, T y)+d(y, T x))
\end{aligned}
$$

for all $x, y \in X$. In 1979, B. E. Rhoades [22] studied a more general form of $\lambda$-generalized contractions (where the terms $d(x, T y)$ and $d(y, T x)$ have different coefficients) in sequentially complete uniform spaces via entourages and the Minkowski's pseudometrics corresponding to them. One can combine Ćirić's and Rhoades' ideas with Jachymski's idea and formulate $G-\lambda$-generalized contractions in metric spaces with a graph as follows:

Let $(X, d)$ be a metric space with a graph $G$. A mapping $T: X \rightarrow X$ is called a $G$ - $\lambda$-generalized contraction if $T$ preserves the edges of $G$ and there exist five functions $a_{1}, a_{2}, a_{3}, a_{4}, a_{5}: X \times X \rightarrow[0, \infty)$ with

$\sup \left\{a_{1}(x, y)+a_{2}(x, y)+a_{3}(x, y)+a_{4}(x, y)+a_{5}(x, y): x, y \in X \times X\right\}=\lambda<1$ such that

$$
\begin{aligned}
d(T x, T y) \leq & a_{1}(x, y) d(x, y)+a_{2}(x, y) d(x, T x)+a_{3}(x, y) d(y, T y) \\
& +a_{4}(x, y) d(x, T y)+a_{5}(x, y) d(y, T x)
\end{aligned}
$$

for all $x, y \in X$ with $(x, y) \in E(G)$.

Now, suppose that $(X, d)$ is a metric space with a graph $G$ and $T: X \rightarrow X$ is a $G-\lambda$ generalized contraction. If $(x, y) \in E(G)$, then an argument similar to that appeared in Example 2.9 establishes that

$$
\begin{aligned}
& d(T x, T y) \leq\left(\sum_{i=1}^{5} a_{i}(x, y)\right) \cdot \max \{d(x, y), d(x, T x), d(y, T y),d(x, T y), d(y, T x)\} \\
& \leq \lambda \cdot \max \{d(x, y), d(x, T x), d(y, T y), d(x, T y), d(y, T x)\},
\end{aligned}
$$

where $a_{1}, a_{2}, a_{3}, a_{4}, a_{5}: X \times X \rightarrow[0, \infty)$ satisfy (2.4). Therefore, $T$ satisfies (Q2) and so $T$ is a $G$-quasi-contraction. Hence every $G$ - $\lambda$-generalized contraction (in particular, every $\lambda$-generalized contraction) is a $G$-quasi-contraction.

2.13. Example. Suppose that $E$ is a nontrivial real Banach space and $P$ is a closed cone in $E$ such that $P \cap(-P)=\{0\}$. It is well-known that $P$ induces a partial order $\preceq_{P}$ on $E$ given by

$$
a \preceq_{P} b \Leftrightarrow b-a \in P \quad(a, b \in E) .
$$

Assume that $d: X \times X \rightarrow E$ is a cone metric on $X$ and $(X, d)$ is a cone metric space (see [12, Definition 1]). In 2010, W.-S. Du [11] showed that if the underlying cone $P$ has nonempty interior and $\xi_{e}: E \rightarrow \mathbb{R}$ is the nonlinear scalarization function defined by

$$
\xi_{e}(a)=\inf \{t \in \mathbb{R}: a \in t e-P\} \quad(a \in E),
$$

where $e$ is an interior point of $P$, then the function $\rho_{e}: X \times X \rightarrow \mathbb{R}$ given by

$$
\rho_{e}(x, y)=\xi_{e}(d(x, y)) \quad(x, y \in X)
$$


defines a metric on $X$, and the natural (cone) topology on $X$ induced by the cone metric $d$ and the metric topology on $X$ induced by the metric $\rho_{e}$ coincide (see [11, Theorems 2.1 and 2.2$])$.

Now, suppose that $T:(X, d) \rightarrow(X, d)$ is a quasi-contraction in the sense of D. Ilic and V. Rakočević [13, Definition 1.2], i.e. there exists a $\lambda \in(0,1)$ such that

$$
d(T x, T y) \preceq_{P} \lambda \cdot u_{x, y}
$$

for all $x, y \in X$ and some

$$
u_{x, y} \in\{d(x, y), d(x, T x), d(y, T y), d(x, T y), d(y, T x)\} .
$$

Suppose further that the underlying cone $P$ has nonempty interior and pick an interior point $e$ of $P$. If $x, y \in X$, since $\xi_{e}$ is positively homogeneous (i.e. $a \in E$ and $t \geq 0$ imply $\left.\xi_{e}(t a)=t \xi_{e}(a)\right)$ and nondecreasing (i.e. $a, b \in E$ and $a \preceq_{P} b$ imply $\xi_{e}(a) \leq \xi_{e}(b)$ ) on $E$ (see [11, Lemma 1.1(v) and (vi)]), it follows that

$$
\begin{aligned}
\rho_{e}(T x, T y) & =\xi_{e}(d(T x, T y)) \\
\leq & \xi_{e}\left(\lambda \cdot u_{x, y}\right) \\
= & \lambda \cdot \xi_{e}\left(u_{x, y}\right) \\
\leq & \lambda \cdot \max \left\{\xi_{e}(d(x, y)), \xi_{e}(d(x, T x)), \xi_{e}(d(y, T y)),\right. \\
& \left.\xi_{e}(d(x, T y)), \xi_{e}(d(y, T x))\right\} \\
= & \lambda \cdot \max \left\{\rho_{e}(x, y), \rho_{e}(x, T x), \rho_{e}(y, T y), \rho_{e}(x, T y), \rho_{e}(y, T x)\right\} .
\end{aligned}
$$

Therefore, $T:\left(X, \rho_{e}\right) \rightarrow\left(X, \rho_{e}\right)$ is also a quasi-contraction and in particular, a $G_{0^{-}}$ quasi-contraction. Hence every quasi-contraction on a cone metric space is a $G_{0}$-quasicontraction whose domain is a suitable metric space with the complete graph $G_{0}$ provided that the underlying cone has nonempty interior.

The following proposition is an immediate consequence of the definition of $G$-quasicontractions and gives a simple procedure to construct new $G$-quasi-contractions from older ones.

2.14. Proposition. Let $(X, d)$ be a metric space with a graph $G$ and $T: X \rightarrow X$ be a mapping.

a) If $T$ preserves the edges of $G$, then $T$ preserves the edges of $G^{-1}$ and $\widetilde{G}$.

b) If $T$ satisfies (Q2) for the graph $G$, then $T$ satisfies (Q2) for both the graphs $G^{-1}$ and $\widetilde{G}$.

c) If $T$ is a $G$-quasi-contraction with a quasi-contractive constant $\lambda \in[0,1)$, then $T$ is both a $G^{-1}$-quasi-contraction and a $\widetilde{G}$-quasi-contraction with a quasi-contractive constant $\lambda$.

To prove the existence of a fixed point for a $G$-quasi-contraction in a complete metric space with a graph, we need some lemmas. The first one is the graph version of [9, Lemma 1] proved by $\mathrm{Lj}$. B. Ćirić and the proof appears here is very similar to Ćirić's proof. Nevertheless, for convenience of the reader, we repeat the detailed proof here.

2.15. Lemma. Let $(X, d)$ be a metric space with a graph $G$ and $T: X \rightarrow X$ be a $G$-quasi-contraction with a quasi-contractive constant $\lambda$. Then

$$
d\left(T^{i} x, T^{j} x\right) \leq \lambda \cdot \operatorname{diam}(O(x ; n)) \quad i, j=1, \ldots, n
$$

for all $x \in C_{T}$ and all $n \in \mathbb{N}$. 
Proof. Let $x \in C_{T}$ and $n \in \mathbb{N}$ be given. If $i$ and $j$ are arbitrary positive integers no more than $n$, then $\left(T^{i-1} x, T^{j-1} x\right) \in E(\widetilde{G})$. By Proposition 2.14(c), $T$ is also a $\widetilde{G}$-quasicontraction with a quasi-contractive constant $\lambda$. In particular, $T$ satisfies (Q2) for the graph $\widetilde{G}$. Therefore,

$$
\begin{aligned}
d\left(T^{i} x, T^{j} x\right)= & d\left(T T^{i-1} x, T T^{j-1} x\right) \\
\leq & \lambda \cdot \max \left\{d\left(T^{i-1} x, T^{j-1} x\right), d\left(T^{i-1} x, T^{i} x\right), d\left(T^{j-1} x, T^{j} x\right),\right. \\
& \left.\quad d\left(T^{i-1} x, T^{j} x\right), d\left(T^{j-1} x, T^{i} x\right)\right\} \\
\leq & \lambda \cdot \operatorname{diam}(O(x ; n)) .
\end{aligned}
$$

The next example shows that both the integers $i$ and $j$ must be positive in Lemma 2.15. In other words, neither $i$ nor $j$ is allowed to be zero.

2.16. Example. Consider the set $\mathbb{R}$ of real numbers with the usual (Euclidean) metric and the complete graph $G_{0}$, and define a mapping $T: \mathbb{R} \rightarrow \mathbb{R}$ by the rule $T x=\frac{x}{2}$ for all $x \in \mathbb{R}$. Then $T$ is a $G_{0}$-quasi-contraction with a quasi-contractive constant $\lambda=\frac{1}{2}$. In addition, $T^{n} x=\frac{x}{2^{n}}$ and $\operatorname{diam}(O(x ; n))=|x|\left(1-\frac{1}{2^{n}}\right)$ for all $x \in \mathbb{R}$ and all $n \in \mathbb{N} \cup\{0\}$. Now, let $x_{0}$ be a positive real number and put $n=2, i=0$ and $j=1$ in Lemma 2.15. Then we have

$$
\left|x_{0}-T x_{0}\right|=\frac{x_{0}}{2}>\frac{x_{0}}{2} \cdot\left(1-\frac{1}{2^{2}}\right)=\lambda \cdot \operatorname{diam}\left(O\left(x_{0} ; 2\right)\right) .
$$

2.17. Lemma. Let $(X, d)$ be a metric space with a graph $G$ and $T: X \rightarrow X$ be a $G$ quasi-contraction. Then for each $x \in C_{T}$ and each $n \in \mathbb{N}$, there exists a positive integer $k$ no more than $n$ such that

$$
\operatorname{diam}(O(x ; n))=d\left(x, T^{k} x\right)
$$

Proof. Let $x \in C_{T}$ and $n \in \mathbb{N}$ be given. If $\operatorname{diam}(O(x ; n))=0$, then $O(x ; n)$ is singleton. In particular, $x$ is a fixed point for $T$ and $d\left(T^{i} x, T^{j} x\right)=0$ for all $i, j=0, \ldots, n$. Thus, the statement holds trivially for any positive integer $k$ no more than $n$.

Otherwise, since $O(x ; n)$ is a finite set, it follows that there exist distinct nonnegative integers $i$ and $j$ no more that $n$ such that $\operatorname{diam}(O(x ; n))=d\left(T^{i} x, T^{j} x\right)$. If both the integers $i$ and $j$ are assumed to be positive, then from Lemma 2.15, we have

$$
\operatorname{diam}(O(x ; n))=d\left(T^{i} x, T^{j} x\right) \leq \lambda \cdot \operatorname{diam}(O(x ; n)),
$$

where $\lambda \in[0,1)$ is a quasi-contractive constant of $T$, a contradiction. Hence either $i$ or $j$ must be zero and the proof is finished.

2.18. Remark. Combining Lemmas 2.15 and 2.17 , one can easily obtain that if $(X, d)$ is a metric space with a graph $G$ and $T: X \rightarrow X$ is a $G$-quasi-contraction with a quasicontractive constant $\lambda$, then for each $x \in C_{T}$ and each $n \in \mathbb{N}$, there exists a positive integer $k$ no more than $n$ such that

$$
d\left(T^{i} x, T^{j} x\right) \leq \lambda \cdot \operatorname{diam}(O(x ; n))=\lambda \cdot d\left(x, T^{k} x\right) \quad i, j=1, \ldots, n .
$$

2.19. Lemma. Let $(X, d)$ be a metric space with a graph $G$ and $T: X \rightarrow X$ be a $G$-quasi-contraction with a quasi-contractive constant $\lambda$. Then

$$
\operatorname{diam}(O(x ; n)) \leq \frac{1}{1-\lambda} \cdot d(x, T x)
$$

for all $x \in C_{T}$ and all $n \in \mathbb{N} \cup\{0\}$. 
Proof. Let $x \in C_{T}$ and $n \in \mathbb{N} \cup\{0\}$ be given. If $n=0$, since $\operatorname{diam}(O(x ; 0))=0$, there remains nothing to prove. Otherwise, from Lemma 2.17, there exists a positive integer $k$ no more than $n$ such that $\operatorname{diam}(O(x ; n))=d\left(x, T^{k} x\right)$. Putting $i=1$ and $j=k$ in Lemma 2.15 , we get

$$
\begin{aligned}
\operatorname{diam}(O(x ; n)) & =d\left(x, T^{k} x\right) \\
& \leq d(x, T x)+d\left(T x, T^{k} x\right) \\
& \leq d(x, T x)+\lambda \cdot \operatorname{diam}(O(x ; n)) .
\end{aligned}
$$

Now the inequality

$$
\operatorname{diam}(O(x ; n)) \leq \frac{1}{1-\lambda} \cdot d(x, T x)
$$

follows immediately.

2.20. Lemma. Let $(X, d)$ be a metric space with a graph $G$ and $T: X \rightarrow X$ be a $G$-quasi-contraction. Then $\left\{T^{n} x\right\}$ is Cauchy for all $x \in C_{T}$.

Proof. Let $x \in C_{T}$ be given. If $m, n \in \mathbb{N}$ and $m \geq n \geq 2$, since $T^{n-1} x \in C_{T}$, it follows that putting $i=m-n+1$ and $j=1$ in Lemma 2.15, we get

$$
d\left(T^{m} x, T^{n} x\right)=d\left(T^{m-n+1} T^{n-1} x, T T^{n-1} x\right) \leq \lambda \cdot \operatorname{diam}\left(O\left(T^{n-1} x ; m-n+1\right)\right),
$$

where $\lambda \in[0,1)$ is a quasi-contractive constant of $T$. Moreover, by Lemma 2.17, there exists a positive integer $k$ no more than $m-n+1$ such that

$$
\operatorname{diam}\left(O\left(T^{n-1} x ; m-n+1\right)\right)=d\left(T^{n-1} x, T^{k+n-1} x\right) .
$$

Because $n \geq 2$, it follows that $T^{n-2} x \in C_{T}$ and so putting $i=1$ and $j=k+1$ in Lemma 2.15 yields

$$
\begin{aligned}
d\left(T^{n-1} x, T^{k+n-1} x\right) & =d\left(T T^{n-2} x, T^{k+1} T^{n-2} x\right) \\
& \leq \lambda \cdot \operatorname{diam}\left(O\left(T^{n-2} x ; m-n+2\right)\right) .
\end{aligned}
$$

Finally, combining (2.6), (2.7) and (2.8), and using induction and Lemma 2.19, we obtain

$$
\begin{aligned}
d\left(T^{m} x, T^{n} x\right) & \leq \lambda \cdot \operatorname{diam}\left(O\left(T^{n-1} x ; m-n+1\right)\right) \\
& =\lambda \cdot d\left(T^{n-1} x, T^{k+n-1} x\right) \\
& \leq \lambda^{2} \cdot \operatorname{diam}\left(O\left(T^{n-2} x ; m-n+2\right)\right) \\
& \vdots \\
& \leq \lambda^{n} \cdot \operatorname{diam}(O(x ; m)) \\
& \leq \frac{\lambda^{n}}{1-\lambda} \cdot d(x, T x) .
\end{aligned}
$$

Letting $m, n \rightarrow \infty$, we find $d\left(T^{m} x, T^{n} x\right) \rightarrow 0$. Hence $\left\{T^{n} x\right\}$ is Cauchy.

Now we are ready to prove our main theorem on the existence of fixed points for $G$-quasi-contractions in complete metric spaces with a graph.

2.21. Theorem. Let $(X, d)$ be a complete metric space with a graph $G$ and $T: X \rightarrow X$ be G-quasi-contraction. Then the restriction of $T$ to $C_{T}$ is a weakly Picard operator if either $T$ is orbitally $\widetilde{G}$-continuous on $X$ or $G$ is a $(\widetilde{\mathrm{C}})$-graph.

In particular, whenever $T$ is orbitally $\widetilde{G}$-continuous on $X$ or $G$ is a $(\widetilde{\mathrm{C}})$-graph, $T$ has a fixed point in $X$ if and only if $C_{T} \neq \emptyset$. 
Proof. If $C_{T}=\emptyset$, then there remains nothing to prove. So assume that $C_{T}$ is nonempty. If $x \in C_{T}$, since $\left(T^{m} x, T^{n} x\right) \in E(\widetilde{G})$ for all $m, n \in \mathbb{N} \cup\{0\}$, it follows that $T x \in C_{T}$. Thus, $C_{T}$ is $T$-invariant, i.e. $T\left(C_{T}\right) \subseteq C_{T}$.

Now, let $x \in C_{T}$ be given. By Lemma 2.20, $\left\{T^{n} x\right\}$ is a Cauchy sequence in $X$ and since $(X, d)$ is complete, there exists an $x^{\star} \in X$ (depending on $x$ ) such that $T^{n} x \rightarrow x^{\star}$. We show that $x^{\star}$ is a fixed point for $T$.

To this end, note first that from $x \in C_{T}$, we have $\left(T^{n} x, T^{n+1} x\right) \in E(\widetilde{G})$ for all $n \in \mathbb{N} \cup\{0\}$. If $T$ is orbitally $\widetilde{G}$-continuous on $X$, then $T^{n} x \rightarrow x^{\star}$ implies $T^{n+1} x=$ $T\left(T^{n} x\right) \rightarrow T x^{\star}$ and by uniqueness of the limit of convergent sequences in metric spaces, we obtain $T x^{\star}=x^{\star}$.

Otherwise, if $G$ is a $(\widetilde{\mathrm{C}})$-graph, since $T^{n} x \rightarrow x^{\star}$, there exists a strictly increasing sequence $\left\{n_{k}\right\}$ of positive integers such that $\left(T^{n_{k}} x, x^{\star}\right) \in E(\widetilde{G})$ for all $k \in \mathbb{N}$. On the other hand, if $\lambda \in[0,1)$ is a quasi-contractive constant of $T$, then by Proposition 2.14(c), $T$ is a $\widetilde{G}$-quasi-contraction with a quasi-contractive constant $\lambda$. In particular, $T$ satisfies (Q2) for the graph $\widetilde{G}$. Therefore,

$$
\begin{aligned}
d\left(T^{n_{k}+1} x, T x^{\star}\right)= & d\left(T T^{n_{k}} x, T x^{\star}\right) \\
\leq \lambda \cdot \max \{ & d\left(T^{n_{k}} x, x^{\star}\right), d\left(T^{n_{k}} x, T^{n_{k}+1} x\right), d\left(x^{\star}, T x^{\star}\right), \\
& \left.d\left(T^{n_{k}} x, T x^{\star}\right), d\left(x^{\star}, T^{n_{k}+1} x\right)\right\}
\end{aligned}
$$

for all $k \in \mathbb{N}$. For a fixed positive integer $k$, one of the five terms appeared in the right side of $(2.9)$ is the maximum. So we consider the following five possible cases:

Case 1: If the first term is the maximum, then

$$
d\left(T^{n_{k}+1} x, T x^{\star}\right) \leq \lambda \cdot d\left(T^{n_{k}} x, x^{\star}\right) ;
$$

Case 2: If the second term is the maximum, then

$$
d\left(T^{n_{k}+1} x, T x^{\star}\right) \leq \lambda \cdot d\left(T^{n_{k}} x, T^{n_{k}+1} x\right) ;
$$

Case 3: If the third term is the maximum, then

$$
\begin{aligned}
d\left(T^{n_{k}+1} x, T x^{\star}\right) & \leq \lambda \cdot d\left(x^{\star}, T x^{\star}\right) \\
& \leq \lambda \cdot\left(d\left(x^{\star}, T^{n_{k}+1} x\right)+d\left(T^{n_{k}+1} x, T x^{\star}\right)\right) .
\end{aligned}
$$

Therefore,

$$
d\left(T^{n_{k}+1} x, T x^{\star}\right) \leq \frac{\lambda}{1-\lambda} \cdot d\left(x^{\star}, T^{n_{k}+1} x\right)=\frac{\lambda}{1-\lambda} \cdot d\left(T^{n_{k}+1} x, x^{\star}\right) ;
$$

Case 4: If the forth term is the maximum, then

$$
\begin{aligned}
d\left(T^{n_{k}+1} x, T x^{\star}\right) & \leq \lambda \cdot d\left(T^{n_{k}} x, T x^{\star}\right) \\
& \leq \lambda \cdot\left(d\left(T^{n_{k}} x, T^{n_{k}+1} x\right)+d\left(T^{n_{k}+1} x, T x^{\star}\right)\right)
\end{aligned}
$$

Therefore

$$
d\left(T^{n_{k}+1} x, T x^{\star}\right) \leq \frac{\lambda}{1-\lambda} \cdot d\left(T^{n_{k}} x, T^{n_{k}+1} x\right) ;
$$

Case 5: Finally, if the fifth term is the maximum, then

$$
d\left(T^{n_{k}+1} x, T x^{\star}\right) \leq \lambda \cdot d\left(x^{\star}, T^{n_{k}+1} x\right)=\lambda \cdot d\left(T^{n_{k}+1} x, x^{\star}\right) .
$$

Clearly, at least one of the above five cases happens for infinitely many indices $k$. Hence $\left\{T^{n_{k}+1} x\right\}$ has a subsequence converging to $T x^{\star}$, and again by the uniqueness of the limit of convergent sequences in metric spaces, we obtain $T x^{\star}=x^{\star}$.

Finally, since $C_{T}$ contains all fixed points of $T$, it follows that $x^{\star} \in C_{T}$. Consequently, $\left.T\right|_{C_{T}}: C_{T} \rightarrow C_{T}$ is a weakly Picard operator. 
Before listing some important consequences of Theorem 2.21, it is worth having a discussion on the hypotheses of Theorem 2.21.

2.22. Remark. In [9, Theorem 1], Lj. B. Cirić has used a weaker type of completeness of metric spaces which had been defined by himself in [8] as follows:

Let $(X, d)$ be a metric space and $T: X \rightarrow X$ be a mapping. The metric space $(X, d)$ is called $T$-orbitally complete if each Cauchy sequence of the iterates of a point of $X$ under $T$ is convergent.

It is clear that every complete metric space $(X, d)$ is $T$-orbitally complete for all mappings $T: X \rightarrow X$, but the converse is not true in general. For instance, the set $\mathbb{Q}$ consisting of all rational numbers with the usual (Euclidean) metric is not a complete metric space whereas $\mathbb{Q}$ is $T$-orbitally complete, where $T: \mathbb{Q} \rightarrow \mathbb{Q}$ is defined by the rule $T x=\frac{x}{2}$ for all $x \in \mathbb{Q}$.

The notion of $T$-orbital completeness of a metric space can be generalized to metric spaces with a graph in several different ways. However, by a subtle look at the proof of Theorem 2.21, it is easily realized that we have only used the following weaker type of $T$-orbital completeness (called, e.g., weak $\widetilde{G}$ - $T$-orbital completeness) in metric spaces with a graph as follows:

Let $(X, d)$ be a metric space with a graph $G$ and $T: X \rightarrow X$ be a mapping. The metric space $(X, d)$ is called "weak $\widetilde{G}$-T-orbitally complete" if for each $x \in$ $C_{T}$, the sequence $\left\{T^{n} x\right\}$ is convergent whenever $\left\{T^{n} x\right\}$ is Cauchy and satisfies $\left(T^{n} x, T^{n+1} x\right) \in E(\widetilde{G})$ for all $n \in \mathbb{N}$.

Obviously, by replacing this new notion with the standard notion of completeness, a new version of Theorem 2.21 is obtained.

2.23. Remark. By a subtle look at the proof of Theorem 2.21 in the case that the mapping $T$ is orbitally $\widetilde{G}$-continuous on $X$, it is easily realized that not the whole but a weaker type of the hypothesis of orbital $\widetilde{G}$-continuity of $T$ is used. Indeed, the sequence $\left\{b_{n}\right\}$ of positive integers in Definition 1.2 is replaced with the sequence $\{n\}$, i.e. the sequence of all positive integers. Using this, a weaker type of orbital $\widetilde{G}$-continuity (called, e.g., weak orbital $\widetilde{G}$-continuity) can be defined as follows:

Let $(X, d)$ be a metric space with a graph $G$. A mapping $T: X \rightarrow X$ is called "weakly orbitally $\widetilde{G}$-continuous" on $X$ if $T^{n} x \rightarrow y$ implies $T^{n+1} x \rightarrow T y$ for all $x, y \in X$ such that $\left(T^{n} x, T^{n+1} x\right) \in E(\widetilde{G})$ for all $n \in \mathbb{N}$.

Obviously, by replacing this new notion with the notion of orbital $\widetilde{G}$-continuity, Theorem 2.21 is strengthened.

Now we present three important consequences of Theorem 2.21 where the graph $G$ is replaced with the special graphs. Firstly, we put $G=G_{0}$ in Theorem 2.21 and we get Ćirić's fixed point theorem [9, Theorem 1] on single-valued quasi-contractions in complete metric spaces instead of $T$-orbitally complete metric spaces as follows:

2.24. Corollary. Every quasi-contraction defined on a complete metric space is a Picard operator.

Proof. Let $(X, d)$ be a complete metric space and $T: X \rightarrow X$ be a quasi-contraction. The set $C_{T}$ is nonempty because $C_{T}=X$. Therefore, by Theorem 2.21, the mapping $T=\left.T\right|_{C_{T}}$ is a weakly Picard operator. In particular, $T$ has a fixed point in $X$. To see that $T$ is a Picard operator, it sufficies to show that $T$ has a unique fixed point in $X$. To this end, suppose that $x^{\star}$ and $x^{\star \star}$ are two fixed points for $T$ in $X$. Then from (2.1) we 
have

$$
\begin{aligned}
& d\left(x^{\star}, x^{\star \star}\right)=d\left(T x^{\star}, T x^{\star \star}\right) \\
& \leq \lambda \cdot \max \{d\left(x^{\star}, x^{\star \star}\right), \underbrace{d\left(x^{\star}, T x^{\star}\right)}_{=0}, \underbrace{d\left(x^{\star \star}, T x^{\star \star}\right)}_{=0}, \\
&\underbrace{d\left(x^{\star}, T x^{\star \star}\right)}_{=d\left(x^{\star}, x^{\star \star}\right)}, \underbrace{d\left(x^{\star \star}, T x^{\star}\right)}_{=d\left(x^{\star \star}, x^{\star}\right)}\} \\
&=\lambda \cdot d\left(x^{\star}, x^{\star \star}\right),
\end{aligned}
$$

where $\lambda \in[0,1)$ is a constant. Hence $d\left(x^{\star}, x^{\star \star}\right)=0$ or equivalently, $x^{\star}=x^{\star \star}$.

2.25. Remark. By a subtle look at the proof of Corollary 2.24, and use an argument similar to that appeared there, we see that both the ends of any link of $G$ cannot be fixed points for a $G$-quasi-contraction, i.e. if $x \neq y, T x=x$ and $T y=y$, then $(x, y) \notin E(G)$. Roughly speaking, no $G$-quasi-contraction can keep both the ends of a link of $G$ fixed. In particular, the following results on the number of the fixed points of $G$-quasi-contractions are obtained:

- No quasi-contraction can have two distinct fixed points.

- If $\preccurlyeq$ is a partial order on $X$, then neither a $G_{1}$-quasi-contraction nor a $G_{2}$-quasicontraction can have two distinct fixed points which are comparable elements of $(X, \preccurlyeq)$.

- If $\varepsilon>0$, then no $G_{3}$-quasi-contraction can have two distinct fixed points which are $\varepsilon$-close elements of $(X, d)$.

Secondly, we consider a partial order on the metric space $(X, d)$ and put $G=G_{1}$ or $G=G_{2}$ in Theorem 2.21. Having done this, the following partially ordered version of Ćirić's fixed point theorem on ordered quasi-contractions in complete metric spaces equipped with a partial order is obtained:

2.26. Corollary. Let $(X, \preccurlyeq)$ be a partially ordered set and d be a metric on $X$ such that $(X, d)$ is a complete metric space. Let $T: X \rightarrow X$ be a mapping which maps comparable elements of $(X, \preccurlyeq)$ onto comparable elements and satisfies $(2.2)$. Then the restriction of $T$ to the set of all points $x \in X$ whose every two iterates under $T$ are comparable elements of $(X, \preccurlyeq)$ is a weakly Picard operator if either $T$ is orbitally $G_{2}$-continuous on $X$ or the triple $(X, d, \preccurlyeq)$ satisfies $(*)$.

In particular, whenever $T$ is orbitally $G_{2}$-continuous on $X$ or the triple $(X, d, \preccurlyeq)$ satisfies (*), $T$ has a fixed point in $X$ if and only if there exists an $x \in X$ such than $T^{m} x$ and $T^{n} x$ are comparable elements of $(X, \preccurlyeq)$ for all $m, n \in \mathbb{N} \cup\{0\}$.

Finally, we put $G=G_{3}$ in Theorem 2.21 and we get the following version of Cirić's fixed point theorem on quasi-contractions in complete metric spaces:

2.27. Corollary. Let $(X, d)$ be a complete metric space and $\varepsilon>0$ be a fixed real number. Let $T: X \rightarrow X$ be a mapping which maps $\varepsilon$-close elements of $(X, d)$ onto $\varepsilon$-close elements and satisfies (2.3). Then the restriction of $T$ to the set of all points $x \in X$ whose every two iterates under $T$ are $\varepsilon$-close elements of $(X, d)$ is a weakly Picard operator.

In particular, $T$ has a fixed point in $X$ if and only if there exists an $x \in X$ such that $T^{m} x$ and $T^{n} x$ are $\varepsilon$-close elements of $(X, d)$ for all $m, n \in \mathbb{N} \cup\{0\}$.

Since Banach $G$-contractions, $G$-Kannan mappings, $G$-Chatterjea mappings, $G$-ĆirićReich-Rus operators, Ćirić-Reich-Rus $G$-contractions and $G$ - $\lambda$-generalized contractions are all a $G$-quasi-contraction, we have also the following fixed point theorem for these contractions as a consequence of Theorem 2.21: 
2.28. Corollary. Let $(X, d)$ be a complete metric space with a graph $G$ and $T: X \rightarrow X$ be a Banach $G$-contraction (a G-Kannan mapping, a G-Chatterjea mapping, a G-ĆirićReich-Rus operator, a Ćirić-Reich-Rus $G$-contraction, or a $G$-A-generalized contraction). Then the restriction of $T$ to $C_{T}$ is a weakly Picard operator if either $T$ is orbitally $\widetilde{G}$ continuous on $X$ or $G$ is a $(\widetilde{\mathrm{C}})$-graph.

In particular, whenever $T$ is orbitally $\widetilde{G}$-continuous on $X$ or $G$ is a $(\widetilde{\mathrm{C}})$-graph, $T$ has a fixed point in $X$ if and only if $C_{T} \neq \emptyset$.

By comparing Corollary 2.28 as a version of Theorem 2.21 for several types of contractions with some recent results in graph metric fixed point theory, one can get the followings:

- If we employ Corollary 2.28 for Banach $G$-contractions, then we obtain a simple and weaker version of $\left[14\right.$, Theorems $3.2\left(4^{\circ}\right)$ and $\left.3.3\left(2^{\circ}\right)\right]$ and [3, Corollary 2];

- If we employ Corollary 2.28 for $G$-Kannan mappings, then we obtain another version of [2, Theorem 3] and [3, Corollary 3] without imposing the assumption of weak $T$-connectedness on the graph (see [3, Definition 8]);

- If we employ Corollary 2.28 for $G$-Chatterjea mappings, then we obtain a new version of Chatterjea's fixed point theorem [5] in complete metric spaces with a graph;

- If we employ Corollary 2.28 for either $G$-Ćirić-Reich-Rus operators or ĆirićReich-Rus $G$-contractions, then we obtain another version of [3, Theorem 6] without imposing the assumption of weak $T$-connectedness on the graph and another version of [6, Theorem 2.2 and Lemma 2.7];

- Finally, if we employ Corollary 2.28 for $G$ - $\lambda$-generalized contractions, then we obtain a new version of [7, Theorem 2.5] and a weaker version of [22, Theorem 1] in complete metric spaces with a graph.

Because convergence of sequences in a cone metric space has already been defined in [12, Definition 2], Picard operators can be generalized naturally from metric to cone metric spaces in the following way:

Let $E$ be a nontrivial real Banach space, $P$ be a closed cone in $E$ such that $P \cap(-P)=\{0\}$, and $(X, d)$ be a cone metric space. A mapping $T: X \rightarrow X$ is called a Picard operator if $T$ has unique fixed point $x^{\star} \in X$ and $T^{n} x \rightarrow x^{\star}$ for all $x \in X$.

Similar to the Cauchy property of sequences in metric spaces and using the idea of formulating convergent sequences in cone metric spaces, the Cauchy property of sequences is defined in cone metric spaces (see [12, Definition 3]). So it is natural to say that a cone metric space is complete if every Cauchy sequence is convergent (see [12, Definition $4]$ ). Hence we have also the following consequence of Corollary 2.24 in complete cone metric spaces where the underlying cone has nonempty interior. This result is another version of [20, Theorem 2.1] and generalizes [12, Theorem 1], [13, Theorem 2.1] and [16, Theorems 2.2 and 2.3].

2.29. Corollary. Every quasi-contraction defined on a complete cone metric space is a Picard operator provided that the underlying cone has nonempty interior.

Proof. Let $E$ be a nontrivial real Banach space, $P$ be a closed cone in $E$ with nonempty interior such that $P \cap(-P)=\{0\}$, and $(X, d)$ be a complete cone metric space. Pick any interior point $e$ of $P$ and consider the metric $\rho_{e}$ given by (2.5). Since the cone metric space $(X, d)$ is complete, it follows from [11, Theorem 2.2(iii)] that the metric space $\left(X, \rho_{e}\right)$ is also complete.

Now, let $T:(X, d) \rightarrow(X, d)$ be a quasi-contraction. As it was shown in Example 2.13, $T:\left(X, \rho_{e}\right) \rightarrow\left(X, \rho_{e}\right)$ is also a quasi-contraction. Therefore, by Corollary 2.24, 
$T:\left(X, \rho_{e}\right) \rightarrow\left(X, \rho_{e}\right)$ is a Picard operator, i.e. $T$ has a unique fixed point $x^{\star} \in X$ and $T^{n} x \rightarrow x^{\star}$ in $\left(X, \rho_{e}\right)$ for all $x \in X$.

On the other hand, it follows from [11, Theorem 2.2(i)] that a sequence $\left\{x_{n}\right\}$ consisting of points of $X$ converges to an $x \in X$ in the cone metric space $(X, d)$ if and only if $\left\{x_{n}\right\}$ converges to the same point $x$ in the metric space $\left(X, \rho_{e}\right)$. Hence $T^{n} x \rightarrow x^{\star}$ in $(X, d)$ for all $x \in X$. Consequently, $T:(X, d) \rightarrow(X, d)$ is a Picard operator.

\section{Acknowledgments}

The authors are thankful to the Payame Noor University for supporting this research and also to the referee for his/her valuable comments to improve the paper.

\section{References}

[1] Aleomraninejad, S.M.A. Rezapour, Sh. and Shahzad, N. Some fixed point results on a metric space with a graph, Topology Appl. 159 (3), 659-663, 2012.

[2] Bojor, F. Fixed points of Kannan mappings in metric spaces endowed with a graph, An. Ştiinţ. Univ. "Ovidius" Constanţa Ser. Mat. 20 (1), 31-40, 2012.

[3] Bojor, F. Fixed point theorems for Reich type contractions on a metric spaces with a graph, Nonlinear Anal. 75 (9), 3895-3901, 2012.

[4] Bondy, J.A. and Murty, U.S.R. Graph theory, (Springer, 2008).

[5] Chatterjea, S.K. Fixed-point theorems, C. R. Acad. Bulgare Sci. 25, 727-730, 1972.

[6] Chifu, C. and Petruşel, G. Generalized contractions in metric spaces endowed with a graph, Fixed Point Theory Appl. 2012:161, 9 pages, 2012.

[7] Ćirić, Lj.B. Generalized contractions and fixed-point theorems, Publ. Inst. Math. (Beograd) (N.S.) 12(26), 19-26, 1971.

[8] Ćirić, Lj.B. On contraction type mappings, Math. Balkanica 1, 52-57, 1971.

[9] Ćirić, Lj.B. A generalization of Banach's contraction principle, Proc. Amer. Math. Soc. 45 267-273, 1974.

[10] Ćirić, Lj.B. Quasi-contractions in Banach spaces, Publ. Inst. Math. (Beograd) (N.S.) 21(35), 41-48, 1977.

[11] Du, W.-S. A note on cone metric fixed point theory and its equivalence, Nonlinear Anal. 72 (5), 2259-2261, 2010

[12] Long-Guang, H. and Xian, Z. Cone metric spaces and fixed point theorems of contractive mappings, J. Nonlinear Anal. Appl. 332 (2), 1468-1476, 2007.

[13] Ilić, D. and Rakočević, V. Quasi-contraction on a cone metric space, Appl. Math. Lett. 22 (5), 728-731, 2009.

[14] Jachymski, J. The contraction principle for mappings on a metric space with a graph, Proc. Amer. Math. Soc. 136 (4), 1359-1373, 2008.

[15] Kadelburg, Z. Pavlović, M. and Radenović, S. Common fixed point theorems for ordered contractions and quasicontractions in ordered cone metric spaces, Comput. Math. Appl. 59 (9), 3148-3159, 2010.

[16] Kadelburg, Z. Radenović, S. and Rakočević, V. Remarks on "Quasi-contraction on a cone metric space", Appl. Math. Lett. 22 (11), 1674-1679, 2009.

[17] Khamsi, M.A. Quasicontraction mappings in modular spaces without $\Delta_{2}$-condition, Fixed Point Theory Appl. 2012, Art. ID 916187, 9 pages.

[18] Petruşel, A. and Rus, I.A. Fixed point theorems in ordered L-spaces, Proc. Amer. Math. Soc. 134 (2), 411-418, 2006.

[19] Ran, A.C.M. and Reurings, M.C.B. A fixed point theorem in partially ordered sets and some applications to matrix equations, Proc. Amer. Math. Soc. 132 (5), 1435-1443, 2004.

[20] Rezapour, Sh. Haghi, R.H. and Shahzad, N. Some notes on fixed points of quasi-contraction maps, Appl. Math. Lett. 23 (4), 498-502, 2010.

[21] Rhoades, B.E. A comparison of various definitions of contractive mappings, Trans. Amer. Math. Soc. 226, 257-290, 1977.

[22] Rhoades, B.E. Fixed point theorems in a uniform space, Publ. Inst. Math. (Beograd) (N.S.) 25(39), 153-156, 1979. 
[23] Rus, I.A. Picard operators and applications, Sci. Math. Jpn. 58 (1), 191-219, 2003. 
